\title{
Influencia de las especies del dosel en la disponibilidad de recursos y regeneración avanzada en un bosque templado lluvioso del sur de Chile
}

\author{
Influence of overstorey species identity on resource availability and variation in \\ composition of advanced regeneration in a temperate rainforest in southern Chile
}

\author{
ALFREDO SALDAÑA ${ }^{1} \&$ CHRISTOPHER H. LUSK
}

${ }^{1}$ Departamento de Botánica, Universidad de Concepción, Casilla 160-C, Concepción, Chile; e-mail para correspondencia: asaldana@udec.cl

\begin{abstract}
RESUMEN
Se ha postulado que la heterogeneidad de hábitat en el sotobosque es un factor que promueve el mantenimiento de la diversidad de especies de la comunidad forestal. En este estudio se evaluó la influencia de cuatro especies dominantes de un bosque templado del sur de Chile, Laureliopsis philippiana, Aextoxicon punctatum, Eucryphia cordifolia y Nothofagus dombeyi, sobre la disponibilidad de recursos y su relación con la composición de la regeneración avanzada. Se estimó el porcentaje de luz difusa, la disponibilidad de nitrógeno, fósforo y calcio, el contenido de nitrógeno, fósforo y calcio de la hojarasca, y se determinó la composición de la regeneración avanzada bajo los doseles de cada una de las cuatro especies. Además, se realizó una ordenación para evaluar la variación de la composición de la regeneración avanzada. Se encontraron diferencias significativas en la transmisión de luz bajo cada especie, así como en el contenido nutricional de la hojarasca y disponibilidad de los nutrientes $\mathrm{N}-\mathrm{NO}_{3}$ y P. Los tres nutrientes evaluados presentaron escasa disponibilidad en el suelo, debido a baja mineralización y alta inmovilización. De acuerdo a la ordenación, la composición de la regeneración avanzada bajo $N$. dombeyi y L. philippiana, fue distinguible de la composición de la regeneración bajo E. cordifolia y A. punctatum. La luz fue el recurso que explicó la mayor parte de la variación en la composición de la regeneración avanzada en el sotobosque. En cambio, la variación en la disponibilidad de nutrientes no tuvo relación con la especie en el dosel. Postulamos que las especies que regeneran bajo este dosel difieren más en sus requerimientos lumínicos que nutricionales, siendo la luz el recurso más limitante en el sotobosque.
\end{abstract}

Palabras clave: bosque siempreverde, composición de la regeneración, mineralización, gradiente de luz.

\begin{abstract}
Understory habitat heterogeneity is believed to be a factor promoting maintenance of species diversity in forest communities. The influence of over storey species identity on resource availability and variation in composition of advanced regeneration was studied in a temperate rain forest in southern Chile. The forest overstorey was dominated by the broadleaved evergreens Laureliopsis philippiana, Aextoxicon punctatum, Eucryphia cordifolia and Nothofagus dombeyi. Availabilities of diffuse light, nitrogen, phosphorous and calcium were measured under these four over storey species, as was nutrient content of leaf litter. Advanced regeneration was sampled in plots beneath each over storey species, and results analyzed by ordination. There were significant differences in light transmission, nutritional content of leaf litter and availability of $\mathrm{N}-\mathrm{NO}_{3}$ and $\mathrm{P}$ beneath the four species. Nevertheless, all nutrients showed low availability due to low mineralization and high immobilization. Ordination results indicate that the composition of advanced regeneration under $N$. dombeyi and L. philippiana differed from the composition of regeneration under A. punctatum or E. cordifolia. The ordination suggested that light level explained a large proportion of compositional variation. We propose that species regenerating in the understory differed more in shade tolerance than in nutritional requirements, light being the most limiting resource in the understorey.
\end{abstract}

Key words: evergreen forest, advanced growth composition, mineralization, light gradient. 


\section{INTRODUCCIÓN}

Se ha planteado que la heterogeneidad en la disponibilidad de recursos es responsable de la mantención de la diversidad vegetal, ya que previene la exclusión competitiva y de este modo promueve la riqueza de especies (Grubb 1977, Ricklefs 1977). Dicha heterogeneidad se ha asociado principalmente al régimen de perturbaciones, de diversa escala espacial y/o temporal (Veblen 1982, White \& Pickett 1985). Sin embargo, las especies arbóreas dominantes también generan micrositios heterogéneos y este fenómeno puede dirigir en mayor o menor grado la dinámica comunitaria (Veblen 1982, White \& Pickett 1985, Canham et al. 1994).

Las especies dominantes del bosque pueden modificar el hábitat y generar variación espacial en la disponibilidad de recursos (Horn 1971, Connell \& Slatyer 1977, Catovsky \& Bazzaz 2000). Este hecho, asociado a las diferencias interespecíficas en los requerimientos de luz y nutrientes de cada especie, conduce a cambios en la composición del bosque (Latham 1992, Pacala et al. 1996, Finzi \& Canham 2000). El éxito en el establecimiento y crecimiento de las especies en un determinado ambiente está estrechamente relacionado con los caracteres fisiológicos y morfológicos asociados a la captura de recursos como luz y nutrientes (Latham 1992, Canham et al. 1994, Le Page et al. 2000). Por consiguiente, la capacidad de las especies de crecer cuando la luz no es limitante, así como de sobrevivir a bajos niveles de luz, es determinante en la dinámica sucesional y en la coexistencia de especies en comunidades forestales (Pacala et al. 1996, Kobe \& Coates 1997).

Por otra parte, en bosques maduros la variación interespecífica en la transmisión de luz desde el dosel hacia el sotobosque (Canham et al. 1999, Frazer et al. 2000), está estrechamente correlacionada con su tolerancia a la sombra (Canham et al. 1994, Aubin et al. 2000). Las especies arbóreas tolerantes a la sombra tienden a presentar copas de mayor profundidad (distancia entre la cima de la copa y el follaje no epicórmico más bajo sensu Canham et al. 1994) y de mayor índice de área foliar que especies intolerantes (Canham et al. 1994).

Las especies dominantes del dosel también pueden influir en la disponibilidad de nutrientes en el suelo bajo sus copas (Finzi et al. 1998a). Existen diferencias interespecíficas en la calidad nutricional de las hojas (Cornelissen \& Thompson 1996, Lusk \& Contreras 1999), y esto determina variación espacial en la disponibilidad de nutrientes en el suelo (Finzi \& Canham 1998, Finzi et al. 1998b, Lambers et al.
1998). En el caso de los bosques nativos del sur de Chile, Pérez (1995) mostró diferencias en las tasas de mineralización de nitrógeno bajo diferentes especies dominantes del dosel.

En el bosque siempreverde chileno se han determinado diferencias interespecíficas en el contenido nutricional de follaje fresco, hojarasca y en la resorción de nitrógeno (Lusk \& Contreras 1999, Lusk et al. 2003). La calidad nutricional del follaje determina la tasa de mineralización y disponibilidad de nutrientes en el suelo. Por otra parte, las especies de este tipo forestal presentaron variación en la profundidad y área foliar de sus copas (Lusk \& Contreras 1999), lo que debe generar diferencias en el ambiente lumínico bajo cada especie del dosel.

De acuerdo a esto, se esperaría encontrar una variación predecible en la disponibilidad de recursos para las plantas que se establecen bajo las especies del dosel, y en consecuencia que la composición de la "regeneración avanzada" (o sea juveniles establecidos bajo el dosel) presente una variación espacial concomitante. El objetivo de este estudio fue determinar el efecto de cuatro especies dominantes del dosel, Laureliposis philippiana (Looser) Schodde, Aextoxicon punctatum R. et P., Eucryphia cordifolia Cav. y Nothofagus dombeyi (Mirb.) Oerst, en la disponibilidad de recursos (luz, N, P y Ca) y en la composición de la regeneración avanzada, en un bosque templado lluvioso del sur de Chile.

\section{MATERIALES Y MÉTODOS}

\section{Sitio de estudio y especies}

Esta investigación se desarrolló en un bosque maduro en el Parque Nacional Puyehue (403' $\mathrm{S}, 7^{\circ} 11^{\prime} \mathrm{O}$; 350-400 $\mathrm{m}$ de altitud). El lugar es de relieve montañoso de levantamiento Terciario, en forma de bloques graníticos $(\mathrm{Su}-$ biabre \& Rojas 1994). Posee clima de montaña, con una precipitación media anual de $3.800 \mathrm{~mm}$ y temperatura media anual es de $8,8{ }^{\circ} \mathrm{C}$. El periodo estival es más benigno con temperatura media de $14,2{ }^{\circ} \mathrm{C}$ y menor pluviosidad (Subiabre \& Rojas 1994). El suelo está constituido por cenizas volcánicas tipo "trumao", depositado sobre rocas granitoides y andesitas (Subiabre \& Rojas 1994). El dosel del bosque se encuentra entre $25-35 \mathrm{~m}$ y está dominado por las especies siempreverdes Laureliopsis philippiana (Looser) Schodde, Aextoxicon punctatum R. et P., Eucryphia cordifolia Cav. y Nothofagus dombeyi (Mirb.) Oerst, sobre $45 \mathrm{~m}$ de altura (Parada et al. 2003). Dichas especies contribuyen al rodal con valores entre un 10 y $40 \%$ del área 
basal total, en los casos de $N$. dombeyi y $L$. philippiana respectivamente (T. Parada \& C. Lusk resultados no publicados). Otras especies presentes en el dosel como Dasyphyllum diacanthoides (Less.) Cabr., Luma apiculata (DC.) Burret y Weinmannia trichosperma Cav., no fueron consideradas en el estudio ya que presentan muy baja abundancia.

\section{Muestreo de regeneración}

En un transecto a través del bosque de $600 \mathrm{~m}$ de largo, en dirección este-oeste, se seleccionaron 15 puntos al azar. Estos fueron separados entre sí por una distancia no menor a $50 \mathrm{~m}$ para reducir el riesgo de repetir individuos y asegurar la independencia de las muestras. En cada punto se eligió un par de árboles conespecíficos de las cuatro especies dominantes del dosel, sin presencia de estrato intermedio. Bajo la copa de cada par de árboles se estableció un cuadrante de muestreo de $3 \mathrm{~m}^{2}$. En cada cuadrante se midió el porcentaje de luz difusa, se determinó la composición de la regeneración avanzada y se estimaron las tasas de mineralización de $\mathrm{N}$ $\mathrm{NO}_{3}{ }^{-}, \mathrm{N}-\mathrm{NH}_{4}+$, $\mathrm{P}$ y Ca ${ }^{++}$.

Se determinó la composición de plantas leñosas entre $50 \mathrm{~cm}$ de altura hasta $2 \mathrm{~m}$, en cuadrantes de $3 \mathrm{~m}^{2}$ bajo las 15 réplicas de cada especie dominante. Con esta información, se efectuó una ordenación de las muestras con el fin de interpretar la composición de estas en términos de la respuesta de las especies a los gradientes ambientales. Esto se desarrolló mediante la técnica de ordenación DCA (análisis de correspondencia sin tendencias).

\section{Porcentaje de luz difusa bajo cada especie del dosel}

Se utilizaron dos sensores cuasi hemisféricos LAI-2000 (LI-COR, Lincoln, Nebraska) para medir el porcentaje de luz difusa e índice de área foliar (IAF) bajo cada par de árboles de las cuatro especies dominantes. Uno de estos instrumentos fue usado para medir a $2 \mathrm{~m}$ de altura sobre cada punto de muestreo y el otro fue ubicado al centro de un claro de aproximadamente $5.000 \mathrm{~m}^{2}$, programado para tomar lecturas a intervalos de $30 \mathrm{~s}$. La estimación de luz difusa es producto de la integración de las lecturas de ambos instrumentos, que equivale al porcentaje de apertura del dosel sobre una campo de visión de $148^{\circ}$. Machado \& Reich (1999) señalan que la medición de apertura del dosel que se obtiene con el LAI-2000 se correlaciona significativamente con la densidad de flujo fotónico fotosintético (PPFD) promedio diario y por consiguiente es una herramienta útil en la evaluación de ambientes de luz.

Tasas de mineralización de $\mathrm{N}-\mathrm{NO}_{3}^{-}, \mathrm{N}-\mathrm{NH}_{4}{ }^{+}, \mathrm{P}$ $y \mathrm{Ca}^{++}$

Las tasas de mineralización de $\mathrm{N}^{-\mathrm{NO}_{3}}{ }^{-}, \mathrm{N}-$ $\mathrm{NH}_{4}{ }^{+}, \mathrm{P} \mathrm{y} \mathrm{Ca}{ }^{++}$se estimaron mediante la diferencia en el contenido inicial y final de incubaciones de suelo, durante un periodo de un mes de verano (enero de 2000). Estas fueron hechas in situ, incubando $50 \mathrm{~g}$ de suelo bajo cada par de árboles de las cuatro especies dominantes. Para esto se extrajo una muestra de suelo de 20 $\mathrm{cm}$ del horizonte $A$ (con un barreno de acero), para luego separar la fracción de suelo orgánica que se incubó in situ en bolsas de polietileno. El análisis del contenido nutricional de las muestras iniciales y finales se determinó, para fósforo disponible, por el método de Olsen mediante extracción con $\mathrm{NaHCO}_{3} \quad 1 \mathrm{M}$ a pH 8.5 (Sadzawka 1990). El nitrógeno disponible (separando $\mathrm{N}-\mathrm{NO}_{3}$ y $\left.\mathrm{N}-\mathrm{NH}_{4}\right)$ se determinó por el método de Kjeldahl (Sadzawka 1990). La determinación de calcio se hizo mediante capacidad de intercambio catiónico, extrayendo con $\mathrm{NH}_{4} \mathrm{Ac}$ a pH 7 (Sadzawka 1990). Estos análisis fueron desarrollados en el Laboratorio de Suelos de la Facultad de Agronomía de la Universidad de Concepción.

\section{Contenido nutricional de la hojarasca}

La hojarasca de cada especie del dosel fue colectada en trampas de $1 \mathrm{~m}^{2}$, puestas a una altura de $40 \mathrm{~cm}$ sobre el suelo. Se dispusieron al azar 10 trampas de hojarasca por parcela en cuatro parcelas de $25 \times 25 \mathrm{~m}$. Estas parcelas no coinciden con los árboles donde se midieron los otros parámetros, pero están localizadas en el mismo sitio de estudio dispuestas para otra investigación. Posteriormente, se separaron cuatro muestras de hojarasca de $10 \mathrm{~g}$ cada una, por especie y por parcela, y se determinó su contenido de N, P y Ca total. El $\mathrm{N}$ total fue determinado por el método de Kjeldahl mediante titulación ácida (Sadzawka 1990); P total por el método de calcinación y colorimetría (Sadzawka 1990); y Ca total por el método de calcinación y absorción atómica (Sadzawka 1990). Estos análisis fueron desarrollados en el Laboratorio de Suelos de la Facultad de Agronomía de la Universidad de Concepción.

\section{Análisis de los datos}

Las diferencias en porcentaje de luz difusa bajo las copas de las cuatro especies del dosel se evaluaron con una ANOVA. Esta prueba también fue 
usada para comparar la concentración de $\mathrm{N}_{\text {total }}$, $\mathrm{P}_{\text {total }}$ y $\mathrm{Ca}_{\text {total }}$ en la hojarasca entre especies. En ambos casos, porcentaje de luz y contenido nutricional de hojarasca, se realizó una prueba de Tukey HSD para comparar entre pares de especies del dosel. Las diferencias en tasas de mineralización de N, P y Ca bajo las especies dominantes se evaluaron con la prueba no paramétrica Kruskal-Wallis, ya que la distribución de la variable dependiente no fue normal y tampoco pudo normalizarse mediante transformación.

Para la ordenación de los micrositios bajo las copas de las cuatro especies dominantes se usó DCA, que ubica las muestras en un espacio de ordenación de menor dimensionalidad, definido por los atributos comunitarios y ambientales de estas, de modo que las muestras más parecidas aparezcan más próximas entre sí y las más diferentes más alejadas (Farrel et al. 1995). Los ejes de ordenación, llamados variables latentes o gradientes, pueden ser combinaciones de varias variables ambientales que expliquen el ordenamiento de las muestras (Farrel et al. 1995). Una vez obtenida la ordenación que extrae los patrones dominantes de la variación en la composición de las muestras, los valores latentes se relacionaron indirectamente a las variables ambientales, mediante la correlación de estos con el porcentaje de luz difusa y disponibilidad de nutrientes.

\section{RESULTADOS}

\section{Niveles de luz bajo las especies del dosel}

El porcentaje de luz difusa bajo las especies del dosel varió entre $2,3 \%$ en $A$. punctatum y $5,8 \%$ en $N$. dombeyi (Fig. 1). Se encontró variación

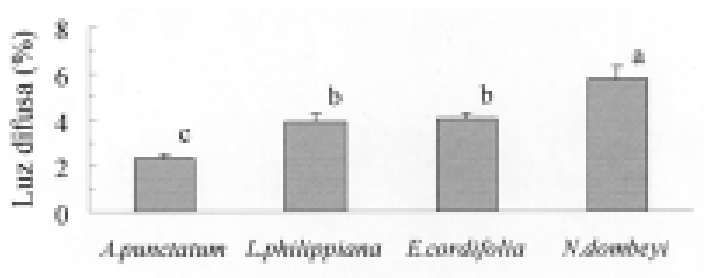

Especies

Fig. 1: Valores medios ( \pm error estándar) de porcentaje de luz difusa bajo las especies dominantes del dosel. Las especies que no presentan diferencia significativa en el contenido del elemento comparten la misma letra $(\mathrm{P}<0,01$; prueba Tukey HSD).

Mean of canopy openness ( \pm standard error) beneath each dominant species. Species that showed no significant differences share the same letter $(\mathrm{P}<0.01$, Tukey HSD test). significativa en el porcentaje de luz difusa bajo las distintas especies (ANOVA, $\mathrm{F}_{3,116}=31,09$; $\mathrm{P}<0,0001)$. Estas diferencias fueron fuertemente significativa entre $A$. punctatum y $N$. dombeyi (prueba Tukey HSD, $\mathrm{P}=0,0002$ ), entre L. philippiana y $N$. dombeyi (prueba Tukey HSD, $\mathrm{P}=0,004)$, y entre E. cordifolia y $N$. dombeyi (prueba Tukey HSD, $\mathrm{P}=0,007$ ).

El índice de área foliar (IAF) mostró diferencias significativas entre las cuatro especies del dosel (ANOVA, $\mathrm{F}_{3,116}=33,14 ; \mathrm{P}<0,0001$ ). El valor promedio de IAF para A. punctatum fue 4,72 $\pm 0,1$, en $L$. philippiana 4,21 $\pm 0,08$, para E. cordifolia $3,97 \pm 0,09$ y en $N$. dombeyi $3,59 \pm 0,08$.

Tasas de mineralización bajo las especies del dosel

La influencia de las especies del dosel sobre la disponibilidad de nutrientes en el suelo bajo las copas de las cuatro especies dominantes mostró que la mineralización en un mes de incubación varió entre $0,6 \mathrm{mg} \mathrm{kg}$ suelo ${ }^{-1}$ en E. cordifolia y $2,8 \mathrm{mg} \mathrm{kg}$ suelo $^{-1}$ en L. philippiana para $\mathrm{N}$ $\mathrm{NO}_{3}{ }^{-}$(Fig. 2) y presentó diferencia significativa entre las cuatro especies del dosel (Tabla 1). En cambio, la diferencia en el contenido inicial y final de $\mathrm{N}^{-} \mathrm{NH}_{4}{ }^{+}$y $\mathrm{P}$ mostró inmovilización bastante pronunciada bajo las cuatro especies del dosel (Fig. 2). Para N-NH${ }_{4}^{+}$esta muestra un rango entre $-17,8 \mathrm{mg} \mathrm{kg}$ suelo $^{-1}$ en E. cordifolia $\mathrm{y}-34,5 \mathrm{mg} \mathrm{kg}$ suelo $^{-1}$ en $A$. punctatum, y no hubo diferencias significativas bajo las especies del dosel. Por su parte la tasa de mineralización de $\mathrm{P}$ varío entre $-4,1 \mathrm{mg} \mathrm{kg}$ suelo $^{-1}$ en $L$. philippiana y $-12,8 \mathrm{mg} \mathrm{kg}$ suelo ${ }^{-1}$ en $A$. punctatum, y mostró diferencias significativas entre las cuatro especies del dosel (Tabla 1). Para $\mathrm{Ca}^{++}$, solo bajo A. punctatum hubo inmovilización (Fig. 2) y bajo las otras tres especies las tasas de mineralización variaron entre $0,9 \mathrm{mg} \mathrm{kg}$ sue$1 \mathrm{o}^{-1}$ en E. cordifolia y $2,3 \mathrm{mg} \mathrm{kg}$ suelo $^{-1}$ en $L$. philippiana, sin embargo estas diferencias no son significativas.

Contenido de N, P y Ca de la hojarasca de las especies del dosel

La hojarasca de las cuatro especies dominantes del dosel mostró diferencias significativas en el contenido nutricional de $\mathrm{N}_{\text {total }}$, $\mathrm{P}_{\text {total }}$ y $\mathrm{Ca}_{\text {total }}$ (Tabla 2). La concentración promedio de $\mathrm{N}_{\text {total }}$ en la hojarasca varió entre $0,4 \mathrm{~g} \mathrm{~g}^{-1}$ en $E$. cordifolia y $0,8 \mathrm{~g} \mathrm{~g}^{-1}$ en L. philippiana (Tabla 3). En la concentración promedio de $\mathrm{P}_{\text {total }}$, el rango fue entre $0,04 \mathrm{~g} \mathrm{~g}^{-1}$ en $E$. cordifolia y $0,12 \mathrm{~g} \mathrm{~g}^{-1}$ en L. Philippiana (Tabla 3). Para $\mathrm{Ca}_{\text {total }}$ el rango 


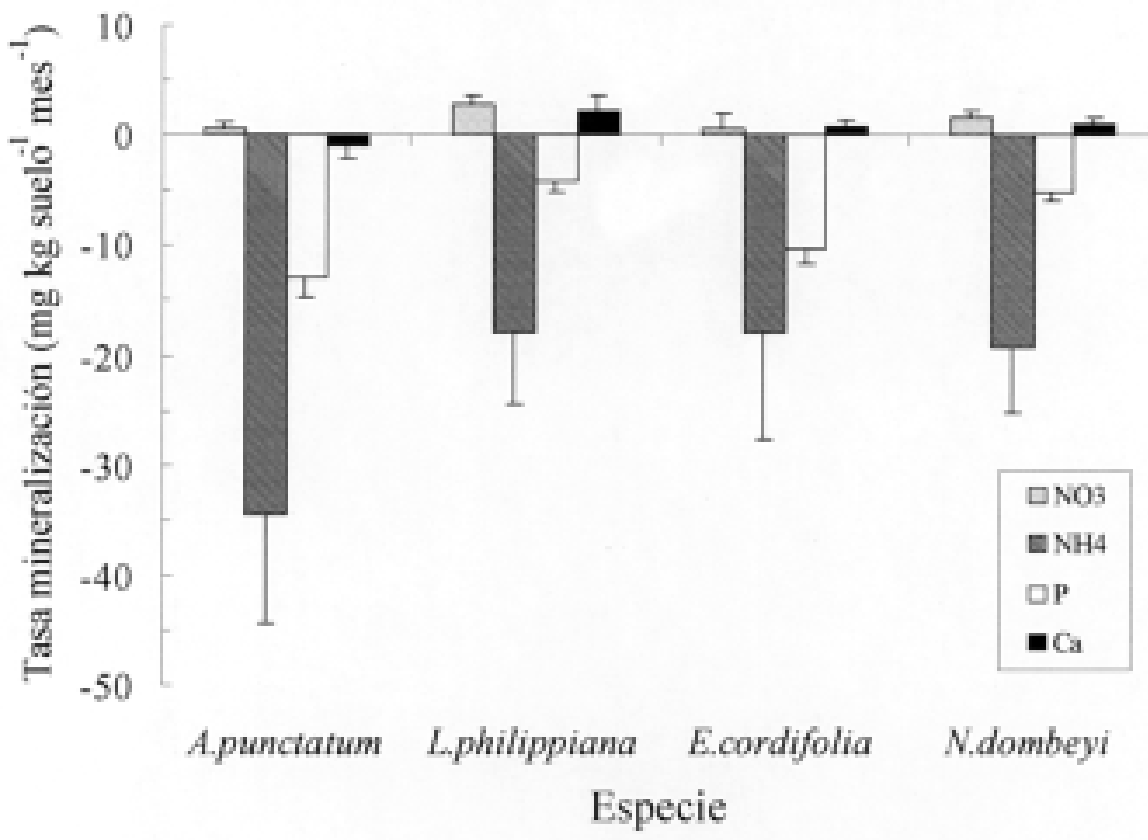

Fig. 2: Tasas de mineralización de $\mathrm{N}_{-} \mathrm{NO}_{3}, \mathrm{~N}-\mathrm{NH}_{4}$, $\mathrm{P}$ y Ca bajo las especies del dosel (mg kg suelo-1 mes $^{-1} \pm$ error estándar).

$\mathrm{N}-\mathrm{NO}_{3}, \mathrm{~N}-\mathrm{NH}_{4}, \mathrm{P}$ and Ca mineralization rates beneath each canopy dominant species (mg kg soil-1 month $^{-1} \pm$ standard error).

TABLA 1

Fuentes de variación, valores de H y P para la prueba de Kruskal-Wallis para la tasa de mineralización de nutrientes bajo las especies dominantes del dosel. Ausencia de significancia estadística se indica como "NS" y efecto significativo se indica como: $\mathrm{P}<0,05(*) ; \mathrm{P}<0,01(* *) ; \mathrm{P}<0,001(* * *)$

Sources of variation, $\mathrm{H}$ and $\mathrm{P}$ values for Kruskal-Wallis on nutrient mineralization rates beneath each dominant canopy species

\begin{tabular}{lccc}
\hline Fuente & Valor de $\mathrm{H}$ & Grados de libertad & Valor de $\mathrm{P}$ \\
\hline $\mathrm{NO}_{3}$ & 10,55 & 3 & $* *$ \\
$\mathrm{NH}_{4}$ & 2,41 & 3 & NS \\
$\mathrm{P}$ & 21,02 & 3 & $* * * *$ \\
$\mathrm{Ca}$ & 4,77 & 3 & $\mathrm{NS}$ \\
\hline
\end{tabular}

TABLA 2

Fuente de variación, valores de F y P para la ANDEVA para la concentración de nutrientes en la hojarasca de las cuatro especies dominantes del dosel. Ausencia de significancia estadística se indica

como "NS" y efecto significativo se indica como: $\mathrm{P}<0,05$ (*) $^{*} \mathrm{P}<0,01(* *) ; \mathrm{P}<0,001(* * *)$

Source of variation, F and $\mathrm{P}$ values for one-way ANOVA on litter nutrient concentrations on each canopy species

\begin{tabular}{lccc}
\hline Fuente & Valor de $\mathrm{H}$ & Grados de libertad & Valor de $\mathrm{P}$ \\
\hline $\mathrm{N}_{\text {total }}$ & 11,96 & 3 & $* * *$ \\
$\mathrm{P}_{\text {total }}$ & 46,54 & 3 & $* * *$ \\
$\mathrm{C}_{\text {atotal }}$ & 55,49 & 3 & $* * *$ \\
\hline
\end{tabular}


de contenido promedio fue entre $1,4 \mathrm{~g} \mathrm{~g}^{-1}$ en $N$. dombeyi y $2,6 \mathrm{~g} \mathrm{~g}^{-1}$ en $A$. punctatum (Tabla 3 ). El contenido nutricional de la hojarasca L. philippiana es superior a la hojarasca de las otras especies analizadas en dos de los tres elementos analizados.

\section{Composición de la regeneración avanzada}

La regeneración avanzada estuvo compuesta por 13 especies y el rango de abundancias relativas varió desde un $27 \%$ en A. punctatum, a un $0,5 \%$ en $W$. trichosperma (Tabla 4). Bajo las cuatro especies del dosel la regeneración avanzada estaba compuesta por casi las mismas cuatro especies, salvo la presencia o ausencia de una o dos especies en cada caso. Los micrositios bajo $N$. dombeyi presentaron regeneración con abundancias relativas homogéneas
(Fig. 3). En cambio bajo A. punctatum las abundancias de Myrceugenia planipes, Caldcluvia paniculata, Dasyphyllum diacanthoides y Rhaphithamnus spinosus fueron mayores que bajo las copas de las otras tres especies del dosel (Fig. 3). Los regeneración bajo L. philippiana y E. cordifolia presentó composiciones y abundancias similares, pero solo bajo las copas de E. cordifolia se encontró individuos juveniles de N. dombeyi (Fig. 3).

\section{Ordenación de la regeneración avanzada bajo las especies del dosel}

La ordenación de la composición muestra que los primeros dos ejes (componentes) explican el $80 \%$ de la variación (Fig. 4). La correlación de los valores latentes de cada componente con el porcentaje de luz difusa y las tasas de mine-

TABLA 3

Concentración media de $\mathrm{N}_{\text {total }}, \mathrm{P}_{\text {total }}$ y $\mathrm{Ca}$ total de la hojarasca de las especies dominantes del dosel ( $\mathrm{g}$ g hojarasca ${ }^{-1} \pm$ error estándar). Las especies que no presentan diferencia significativa en el contenido del elemento comparten la misma letra ( $\mathrm{P}<0,05$; prueba de Tukey HSD)

Mean litter N, P and Ca concentrations of each canopy species ( $\mathrm{g} \mathrm{g}$ litter ${ }^{-1} \pm$ error standard). Species which did not showed significative difference shared the same letter $(\mathrm{P}<0.05$; Tukey HSD test $)$

\begin{tabular}{llllr}
\hline & A. punctatun & L. philippiana & E. cordifolia & N. dombeyi \\
\hline $\mathrm{N}_{\text {total }}$ & $0,61 \pm 0,13^{\mathrm{b}}$ & $0,84 \pm 0,06^{\mathrm{a}}$ & $0,44 \pm 0,04^{\mathrm{b}}$ & $0,54 \pm 0,06^{\mathrm{b}}$ \\
$\mathrm{P}_{\text {total }}$ & $0,11 \pm 0,03^{\mathrm{a}}$ & $0,12 \pm 0,01^{\mathrm{a}}$ & $0,04 \pm 0,01^{\mathrm{b}}$ & $0,06 \pm 0,01^{\mathrm{b}}$ \\
$\mathrm{Ca}_{\text {total }}$ & $2,64 \pm 0,29^{\mathrm{a}}$ & $2,00 \pm 0,07^{\mathrm{b}}$ & $2,19 \pm 0,08^{\mathrm{b}}$ & $1,43 \pm 0,03^{\mathrm{c}}$ \\
\hline
\end{tabular}

TABLA 4

Listado de especies que componen la regeneración avanzada bajo las cuatro especies dominantes y sus abundancias relativas totales

Composition and abundance of advanced regeneration beneath the four dominant canopy species

\begin{tabular}{lccc}
\hline Especie & Código & $\begin{array}{c}\text { Abundancia } \\
\text { (\% del total) }\end{array}$ & $\begin{array}{c}\text { Requerimientos lumínicos(*) } \\
\text { (\% luz difusa) }\end{array}$ \\
\hline Amomyrtus luma (Mol.) Legr, et Kausel & & 6,8 & 8 \\
Aextoxicon punctatum R. et P. & AL & 26,9 & 2 \\
Azara serrata R. et P. & AP & 3,6 & 10 \\
Caldcluvia paniculata (Cav.) D. Don & AZ & 9,4 & 2 \\
Dasyphyllum diacanthoides (Less.) Cabr. & CP & 9,6 & 9 \\
Eucryphia cordifolia Cav. & DD & 7,2 & 9 \\
Gevuina avellana Mol. & EC & 0,8 & 10 \\
Luma apiculata (DC.) Burret & GA & 2 & 7 \\
Laureliposis philippiana (Looser) Schodde & LA & 3,3 & 4 \\
Myrceugenia planipes (H. et A.) Berg & MP & 16 & $<1$ \\
Nothofagus dombeyi (Mirb.) Oerst. & ND & 4,3 & 37 \\
Rhaphithamnus spinosus (Juss.) Mold. & RS & 9,5 & 6 \\
Weinmannia trichosperma Cav. & WT & 0,5 & 13 \\
\hline
\end{tabular}

(*) Ex: Lusk \& Kelly (2003) 


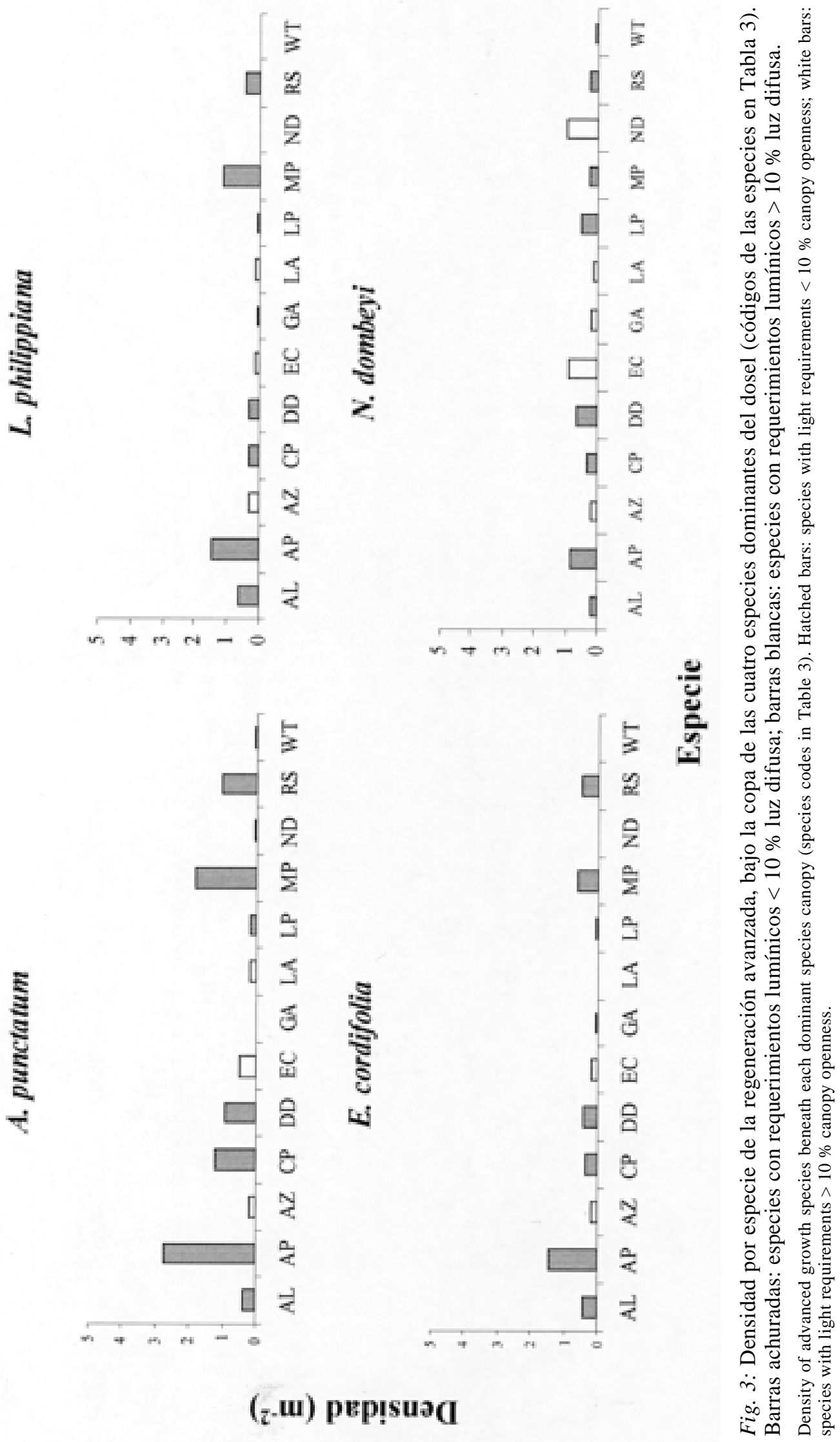




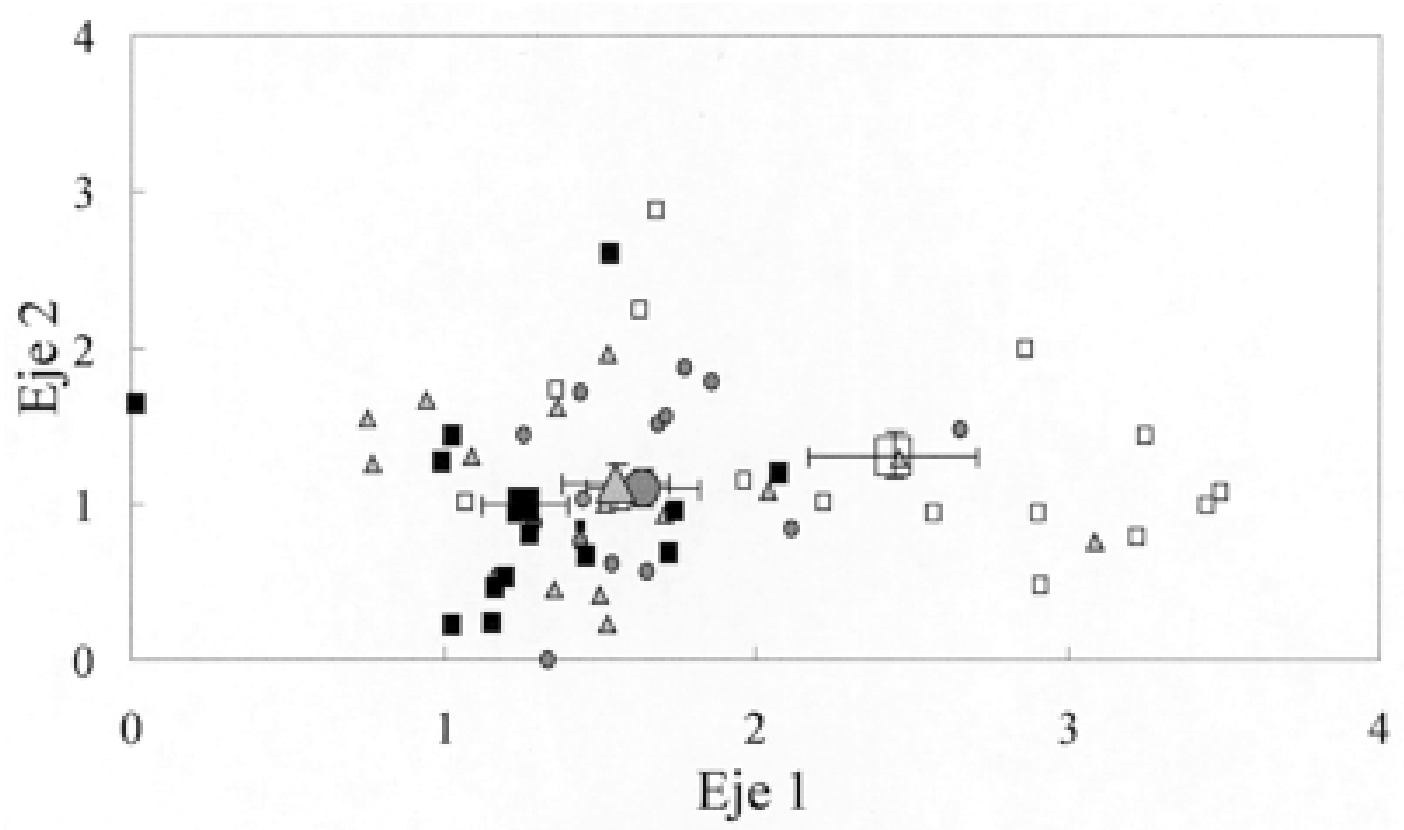

Fig. 4: Ordenación de los micrositios bajo la copas de A. punctatum (O), L. philippiana (ם), E. cordifolia $(\mathbf{\Delta})$ y $N$. dombeyi $(\square)$, en base a la composición de la regeneración avanzada. La ubicación de cada micrositio se muestra en los símbolos pequeños. Los símbolos grandes corresponden al promedio de los valores de ambos ejes (centroide, intervalo de confianza $95 \%$ ) para cada especie.

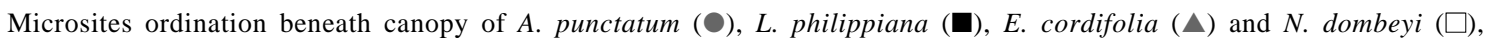
based on advanced growth composition. Small symbols represent each miscrosite. Large symbols represent mean value of both axes for each canopy species.

ralización de cada replica (Tabla 5) indicaron que la variación en composición de regeneración fue explicada por el gradiente lumínico asociado al primer componente (Eigenvalor $=$ $0,53)$, y en menor grado por el gradiente de disponibilidad de $\mathrm{N}^{-\mathrm{NO}_{3}}{ }^{-}$y $\mathrm{P}$, asociado al segundo componente $($ Eigenvalor $=0,31)$.

La composición de la regeneración avanzada bajo las copas de las cuatro especies dominantes del dosel no mostró una asociación clara con el eje correlacionado con el gradiente de disponibilidad de nutrientes, pero sí con el eje asociado al gradiente lumínico. Las muestras que, en base a su composición y abundancias, se agrupan en la parte del gradiente con mayor disponibilidad de luz, corresponden a la regeneración avanzada bajo el dosel de $N$. dombeyi. En cambio la composición de la regeneración avanzada bajo $L$. philippiana, se ubican en la zona del gradiente lumínico de menor disponibilidad.

La ordenación de las especies que componen la regeneración avanzada, del total de micrositios, bajo las cuatro especies dominantes del dosel, posee los mismos eigenvalores para cada componente que la ordenación anterior (arriba). De estas especies, las que presentaron las mayores abundancias relativas, se ubican de la mitad hacia abajo del gradiente de disponibilidad de luz definido por el primer eje. Los valores latentes de cada especie en esta ordenación, no presentaron correlación con los valores de luz difusa o disponibilidad de nutrientes medidos bajo las copas de las especies dominantes. Sin embargo, los valores latentes del primer eje muestran una correlación positiva significativa con los requerimientos de luz de los juveniles de cada especie $\left(\mathrm{R}^{2}=0,52 ; \mathrm{F}_{1,10}=\right.$ 2,20; $\mathrm{P}<0,5$ ) (Fig. 5). Los requerimientos de luz corresponden a los valores de luz difusa en los sitios en que se detectaron las densidades máximas de plántulas y brinzales en el sotobosque para cada especie que compone la regeneración (Lusk \& Kelly 2003).

\section{DISCUSIÓN}

\section{Disponibilidad de recursos}

La influencia de las cuatro especies dominantes del dosel determinó una variación en la disponibilidad de recursos como luz y nutrientes en 
TABLA 5

Correlación de los valores latentes de cada componente de la ordenación con las variables luz difusa $(\%)$ y tasa de mineralización $\left(\mathrm{mg} \mathrm{kg} \mathrm{suelo}{ }^{-1} \mathrm{mes}^{-1}\right)$

Correlation between each component ordination values and resource availability

\begin{tabular}{lcc}
\hline Variable & Eje 1 & Eje 2 \\
\hline Luz $(\%)$ & $\mathrm{R}^{2}=0,1 ; \mathrm{F}_{1,58}=5,29 ; \mathrm{P}=0,02$ & $\mathrm{NS}$ \\
$\mathrm{N}^{-\mathrm{NO}_{3}}$ & $\mathrm{NS}$ & $\mathrm{R}^{2}=-0,2 ; \mathrm{F}_{1,58}=8,94 ; \mathrm{P}=0,004$ \\
$\mathrm{~N}^{-\mathrm{NH}_{4}}$ & $\mathrm{NS}$ & $\mathrm{NS}$ \\
$\mathrm{P}$ & $\mathrm{NS}$ & $\mathrm{R}^{2}=-0,1 ; \mathrm{F}_{1,58}=4,2 ; \mathrm{P}=0,04$ \\
$\mathrm{Ca}$ & $\mathrm{NS}$ & $\mathrm{NS}$ \\
\hline
\end{tabular}

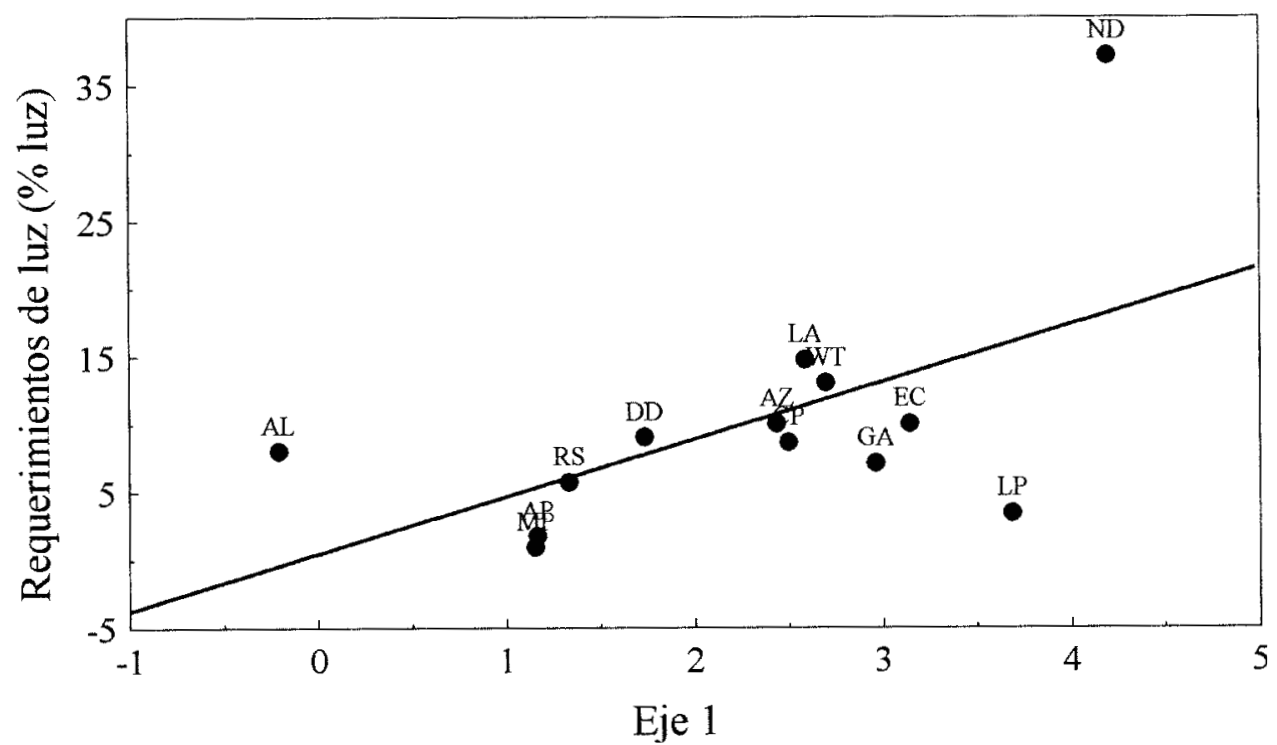

Fig. 5: Relación entre los valores latentes del primer eje de la ordenación de especies que componen la regeneración y sus requerimientos de luz (valores en Tabla 4).

Relationship between first axes species ordination values and them light requirements.

el sotobosque. Esta variación puede ser relacionada con las distintas características fisiológicas, morfológicas y arquitectónicas que presentan dichas especies.

Los niveles de luz difusa bajo el dosel difirieron significativamente entre las cuatro especies (Fig. 1) en correspondencia con sus diferencias en sombratolerancia. La especie definida como más tolerante entre las cuatro $(A$. punctatum), presentó menor transmisión de luz hacia el sotobosque. En cambio, el mayor nivel lumínico se registró bajo $N$. dombeyi, especie que es la menos sombratolerante de las cuatro (Donoso 1981, Lusk \& Kelly 2003). Se ha descrito una estrecha relación entre el nivel de to- lerancia a la sombra de especies arbóreas y la transmisión de luz hacia al sotobosque (Canham et al. 1994). Resultados del presente estudio coinciden con este hecho, ya que A. punctatum y L. philippiana poseen copas con mayor índice de área foliar que $N$. dombeyi.

Por otra parte, la disponibilidad de nutrientes también depende de las especies del dosel, ya que bajo las copas se encontraron diferencias interespecíficas en la tasa de mineralización neta de nutrientes. Las condiciones nutritivas más favorables se presentaron bajo $L$. philippiana, donde $\mathrm{N}, \mathrm{P}$ y Ca estaban más disponibles que bajo las otras especies (Fig. 2). En cambio, bajo A. punctatum la disponibilidad de 
nutrientes fue mucho menor, dadas las menores tasas de mineralización netas. Dicha variación puede ser explicada en parte por diferencias en la calidad nutritiva de la hojarasca entre las especies, tanto en este estudio (Tabla 3), como en otros reportes para las mismas especies (Pérez 1995, Lusk \& Contreras 1999). La calidad de la hojarasca influye en su tasa de descomposición disponibilidad de los nutrientes mineralizados (Cornelissen \& Thompson 1996, Lambers et al. 1998). Sin embargo, no solo la calidad nutritiva del follaje determinaría la tasa de descomposición y mineralización sino que también el área foliar específica (AFE) de las hojas. Las hojas de menor AFE son más gruesas y/o densas y se descomponen más lentamente que las hojas de mayor AFE (Lusk et.al. 2001). Esto explicaría la baja disponibilidad de nutrientes bajo $A$. punctatum, que aunque posee hojarasca de calidad nutricional superior a $E$. cordifolia o $N$. dombeyi (Tabla 3), presentó un valor de AFE menor a las otras especies (Lusk 2002).

La concentración de nitratos $\left(\mathrm{N}-\mathrm{NO}_{3}{ }^{-}\right)$fue cercana a cero, lo que reflejaría el nivel residual de este compuesto en estos suelos, debido a su escasa mineralización (Fig. 2). Aunque no se encontraron diferencias significativas en la inmovilización de algunos nutrientes como $\mathrm{N}$ $\mathrm{NH}_{4}{ }^{+}$y Ca ${ }^{++}$, la magnitud de los valores promedios bajo cada especie muestra una tendencia dominante de significancia marginal (Fig. 2). El hecho de que la mayor parte de los nutrientes se encuentren inmovilizados bajo las cuatro especies dominantes, puede estar asociado a los factores que limitan la descomposición de la materia orgánica entre micrositios, y en consecuencia que determinan la mineralización y disponibilidad de nutrientes en el suelo (Perry 1994). Tian et al. (1992) señalan que la razón $\mathrm{C}: \mathrm{N}$ y el contenido de polifenoles y lignina de las hojas afectan la descomposición, ya que la mineralización de hojarasca de 10 especies forestales aumentó con el contenido de $\mathrm{N}$ foliar $\mathrm{y}$ disminuyó con los contenidos de lignina y polifenoles. En el presente estudio, probablemente estas características de la hojarasca determinaron que la mayor parte del $\mathrm{N}$ y el $\mathrm{P}$ estén inmovilizados. Además, el $\mathrm{pH}$ del suelo es ácido ( $\mathrm{pH} 4,5-5,0)$ lo cual puede contribuir a que la descomposición y nitrificación sean más lentas (Donnelly et al. 1990). Por qué bajo el dosel existe una fuerte inmovilización, sería útil integrar al análisis la composición química de la hojarasca y no solo la concentración de N, P y $\mathrm{Ca}$. El hecho de que se encuentre más inmovilización que mineralización, es una tendencia que se ha descrito para bosques templados (Vitousek 1984b) y además se considera un meca- nismo importante de conservación de nutrientes del sitio (Perry 1994).

\section{Respuesta de la composición de la regenera- ción avanzada}

Las especies dominantes del dosel influyeron sobre la composición de la regeneración avanzada. Esta influencia fue mayor bajo $N$. dombeyi y L. philippiana, pues estas especies presentaron diferencias en composición y abundancia de la regeneración comparadas con los sitios bajo E. cordifolia y A. punctatum, en el diagrama de ordenación (Fig. 4). Este patrón podría atribuirse a la influencia de las especies del dosel sobre la disponibilidad de recursos. Otros estudios han mostrado diferencias interespecíficas en las tasas de crecimiento y mortalidad de plántulas o juveniles en respuesta a la variación en los niveles de recursos (Latham 1992, Finzi \& Canham 2000), de manera que estas variaciones alterarían la abundancia relativa por especie y su probabilidad de dominar el bosque (Pacala et al. 1994).

La regeneración avanzada estuvo compuesta por especies que difieren en sombratolerancia (Donoso 1981, Lusk \& Kelly 2003) y las mayores abundancias relativas las presentaron aquellas especies que han sido definidas como más tolerantes a la sombra (Fig. 3). Los valores latentes de la ordenación de especies que componen la regeneración, presentaron una correlación positiva significativa con los requerimientos lumínicos determinados para cada una por Lusk \& Kelly (2003), lo que explicaría las variaciones en composición de la regeneración avanzada asociadas a la luz.

El nivel lumínico bajo las copas de los árboles fue el recurso que explicó la mayor parte de la variación en la composición de la regeneración. Este factor estuvo asociado significativamente al gradiente definido por el primer eje de ordenación (Fig. 4, Tabla 5). En cambio, la variación en la disponibilidad de nutrientes bajo las copas no afectó la composición de la regeneración de manera discernible. Dicha variación limitaría menos el crecimiento de plántulas y juveniles de especies arbóreas que la disponibilidad de luz en otros bosques templados, especialmente los de dosel caducifolio ( $\mathrm{Pa}$ cala et al. 1994, Kobe et al. 1995, Finzi \& Canham 2000).

Durante la sucesión el dosel del bosque va desde una organización abierta y agrupada, a un dosel cerrado y uniforme que además presenta una estructura vertical más compleja producto del autorraleo y muerte de árboles (Brown \& Parker 1994). Este cambio estructu- 
ral determina variación en la disponibilidad de luz en el sotobosque. En el bosque templado lluvioso chileno, Aravena et al. (2002) encontraron que a través de una secuencia cronológica, la riqueza de plántulas y brinzales de especies arbóreas no disminuye en un rodal antiguo. Por lo tanto, dado que nuestros resultados muestran que la disponibilidad de luz es el recurso que explica la mayor variación en composición de la regeneración avanzada, la influencia de las especies que dominan el dosel es clave en la variación lumínica que promueve la mantención de riqueza de especies.

\section{AGRADECIMIENTOS}

Este trabajo es parte de la tesis para obtener el grado de Magíster en Ciencias mención Botánica en la Universidad de Concepción. Agradecemos los comentarios del Dr. Lohengrin Cavieres de la Universidad de Concepción, y al Dr. Francisco Matus de la Universidad de Talca. Esta investigación fue financiada por el proyecto FONDECYT 1000367.

\section{LITERATURA CITADA}

ARAVENA JC, M CARMONA, C PÉREZ \& J ARMESTO (2002) Changes in tree species richness and soil properties in a successional chronosequence in northern Chiloé Island, Chile. Revista Chilena de Historia Natural 75: 339-360.

AUBIN I, M BEAUDET \& C MESSIER (2000) Light extinction coefficients specific to the understory vegetation of the southern boreal forest, Quebec. Canadian Journal of Forest Research 30: 168-177.

BROWN M \& G PARKER (1994) Canopy light transmittance in a chronosequence of mixed-species deciduous forests. Canadian Journal of Forest Research 24: 1694-1703.

CANHAM C, A FINZI, S PACALA \& D BURBANK (1994) Causes and consequences of resource heterogeneity in forests: interspecific variation in light transmission by canopy trees. Canadian Journal of Forest Research 24: 337-349.

CANHAM C, D COATES, P BARTEMUCCI \& $S$ QUAGLIA (1999) Measurement and modeling of spatially explicit variation in light transmission through interior cedar-hemlock forests of British Columbia. Canadian Journal of Forest Research 29: 1775-1783.

CATOVSKY S \& F BAZZAZ (2000) The role of resource interactions and seedling regeneration in maintaining a positive feedback in hemlock stand. Journal of Ecology 88: 100-112.

CONNELL J \& R SLATYER (1977) Mechanism of succession in natural communities and their role in community stability and organization. American Naturalist 111: 1119-1144.

CORNELISSEN J \& K THOMPSON (1996) Functional leaf attributes predict litter decomposition rate in herbaceous plants. New Phytologist 127: 109-114.
DONNELLY PK, JA ENTRY, DL CRAWFORD \& JR CROMACK K (1990) Cellulose and lignin degradation in forest soils: response to moisture, temperature, and acidity. Microbial Ecology 20: 289-295

DONOSO C (1981) Ecología forestal: el bosque y su medio ambiente. Editorial Universitaria, Santiago, Chile. 368 pp.

FARREL E, A CRITCHLEY \& L CID (1995) Análisis multivariado de comunidades de algas. En: Alveal K, M Ferrario, E Oliveira \& E Sar (eds) Manual de métodos ficológicos: 795-823. Ediciones Universidad de Concepción, Concepción, Chile.

FRAZER G, J TROFYMOW \& K LERTZMAN (2000) Canopy openness and leaf area in chronosequences of coastal temperate rainforests. Canadian Journal of Forest Research 30: 239-256.

FINZI A \& C CANHAM (1998) Non-additive effects of litter mixtures on net $\mathrm{N}$ mineralization in a southern New England forest. Forest Ecology and Management 105: 129-136.

FINZI A, N VAN BREEMEN \& C CANHAM (1998a) Canopy tree-soil interactions within temperate forests: species effects on soil carbon and nitrogen. Ecological Applications 8: 440-446.

FINZI A, C CANHAM \& N VAN BREEMEN (1998b) Canopy tree-soil interactions within temperate forests: species effects on $\mathrm{pH}$ and cat ions. Ecological Applications 8: 447-454.

FINZI A \& C CANHAM (2000) Sapling growth in response to light and availability in a southern New England forest. Forest Ecology and Management 131: 153-165.

GRUBB P (1977) The maintenance of species richness in plant communities: the importance of the regeneration niche. Biological Reviews 52: 107-145.

HORN H (1971) The adaptive geometry of trees. Princeton University Press, Princeton, New Jersey, USA. 114 pp.

KOBE R, S PACALA, J SILANDER \& C CANHAM (1995) Juvenile tree survivorship as a component of shade tolerance. Ecological Applications 5: 517-532.

KOBE R \& K COATES (1997) Models of sapling mortality as a function of growth characterize interspecific variation in shade tolerance of eight tree species of northwestern British Columbia. Canadian Journal of Forest Research 27: 227-236.

LAMBERS H, F CHAPIN \& T PONS (1998) Plant physiological ecology. Springer-Verlag, New York, New York, USA. 540 pp.

LATHAM R (1992) Co-occurring tree species change rank in seedling performance with resourced varied experimentally. Ecology 73: 2129-2144.

LE PAGE P, C CANHAM, D COATES \& $P$ BARTEMUCCI (2000) Seed abundance versus substrate limitation of seedling recruitment in northern temperate forests of British Columbia. Canadian Journal of Forest Research 30: 415-427.

LUSK C (2002) Leaf area accumulation helps juvenile evergreen trees tolerate shade in a temperate rainforest. Oecologia 132: 188-196.

LUSK C \& O CONTRERAS (1999) Foliage area and crown nitrogen turnover in temperate rain forest juvenile trees of differing shade tolerance. Journal of Ecology 87: 973-983.

LUSK C \& CK KELLY (2003) Interspecific variation in seed size and safe sites in a temperate rain forest. New Phytologist 158: 535-541.

LUSK C, C DONOSO, M JIMÉNEZ, C MOYA, G OYARCE, R REINOSO, A SALDAÑA, P VILLEGAS \& F MATUS (2001) Descomposición 
de hojarasca de Pinus radiata y tres especies arbóreas nativas. Revista Chilena de Historia Natural 74: 705-710.

LUSK C, MATUS F, MORENO-CHACÓN M, SALDAÑA A \& M JIMÉNEZ-CASTILLO (2003) Seasonal variation in leaf litter nutrient concentrations of Valdivian rainforest trees. Gayana Botánica (Chile) 60: 35-39.

MACHADO J \& P REICH (1999) Evaluation of canopy openness as predictors of photosynthetic flux density in deeply shaded conifer-dominated forest understory. Canadian Journal of Forest Research 29: 1438-1444.

PACALA S, C CANHAM, J SILANDER \& R KOBE (1994) Sapling growth as a function of resources in a northern temperate forest. Canadian Journal of Forest Research 24: 2172-2183.

PACALA S, C CANHAM C, J SAPONARA, J SILANDER, R KOBE \& E RIBBENS (1996) Forest models defined by field measurements: estimation, error analysis, and dynamics. Ecological Monographs 66: 1-43.

PARADA T, JARA C \& C LUSK (2003) Distribución de alturas máximas de especies en rodales antiguos de selva valdiviana. Bosque (Chile) 24: 63-67.

PÉREZ C (1995) Los procesos de descomposición de la materia orgánica de bosques templados costeros: interacción entresuelo, clima y vegetación. En: Armesto J, C Villagrán \& MK Arroyo (eds)

Editor Asociado: Juan Armesto

Recibido el 17 de abril de 2002; aceptado el 4 de julio de 2003
Ecología de los bosques nativos de Chile: 301-313. Editorial Universitaria, Santiago, Chile.

PERRY D (1994) Forest ecosystems. The Johns Hopkins University Press, Baltimore, Maryland, USA. 649 pp.

RICKLEFS RE (1977) Environmental heterogeneity and plant species diversity: a hypothesis. American Naturalist 111: 376-381.

SADZAWKA A (1990) Métodos de análisis de suelos. Estación Experimental La Platina, Santiago, Chile. 256 pp.

SUBIABRE A \& C ROJAS (1994) Geografía física de la región de los lagos. Ediciones Universidad Austral de Chile, Valdivia, Chile. 118 pp.

TIAN G, B KANG \& L BRUSSAARD (1992) Effects of chemical composition on $\mathrm{N}, \mathrm{CA}$ and $\mathrm{Mg}$ release during incubaction of leaves from selected agroforestry and fallow plant species. Biogeochemistry 16: 103-119.

VEBLEN T (1982) Growth patterns of Chusquea bamboos in the understory of Chilean Nothofagus forests and their influences in forests dynamics. Bulletin of the Torrey Botanical Club 109: 474-487.

VITOUSEK P (1984b) Litterfall, nutrient cycling, and nutrient limitation in tropical forests. Ecology 65 : 285-298.

WHITE P \& T PICKET (1985) Natural disturbance and patch dynamics: an introduction. En: Picket $\mathrm{T} \& \mathrm{P}$ White (eds) The ecology of natural disturbance patch dynamics. Academic Press Inc., London, United Kingdom. 470 pp. 